\title{
DESMÍDIAS DE AMBIENTES FITOTÉLMICOS BROMELÍCOLAS
}

\author{
SOPHIA, M. G. \\ Laboratório de Ficologia, Departamento de Botânica, Museu Nacional, UFRJ, Quinta da Boa Vista, São Cristóvão, \\ CEP 20940-040, Rio de Janeiro, RJ, Brasil. \\ Correspondência para: Maria da Graça Sophia, Departamento de Botânica, Museu Nacional, UFRJ, Quinta da Boa Vista, São \\ Cristóvão, CEP 20940-040, Rio de Janeiro, RJ, Brasil. \\ Recebido em 13/04/98 - Aceito em 24/07/98 - Distribuído em 23/02/99
}

(Com 36 figuras)

\begin{abstract}
Desmids of phytothelmic environments

Water is accumulated in phytotelmata (plant pond) of Bromeliads and usually favor algal development. Twenty-five samples were taken in these environments placed on coastal and mountain regions of Rio de Janeiro State, Brasil. Desmids were dominant in nineteen of these samples. Ten species, three varieties and one taxonomic form were identified. In addition, representatives of Chlorophyceae, Cyanophyceae, Bacillariophyceae, Xanthophyceae and Dinophyceae were found.
\end{abstract}

Key words: agae, desmids, phytothelm, Southeast of Brasil.

\section{RESUMO}

Pela arrumação de suas folhas as bromeliáceas permitem a retenção de água nos imbricamentos das mesmas, onde é possível o desenvolvimento de uma ficoflórula associada aos fitotelmatas (tanques de bromélias). Nestes ambientes, foram coletadas ao acaso vinte e cinco amostras através do Estado do Rio de Janeiro. Deste total, dezenove continham representantes de desmídias. A flora fitotélmica de desmídias esteve representada por quatorze táxons, tendo sido identificadas dez espécies, três variedades e uma forma taxonômica. Além do predomínio das Zygnemaphyceae, foram também registradas outras cinco classes de algas neste tipo de ambiente: Chlorophyceae, Cyanophyceae, Bacillariophyceae, Xanthophyceae e Dinophyceae.

Palavras-chave: algas, desmídias, fitotelmo, Sudeste do Brasil.

\section{INTRODUÇÃO}

Segundo Hadel (1989) o termo fitotelmata foi criado por Varga (1928) e reutilizado por Maguire (1971), referindo-se aos microecossistemas aquáticos mantidos por estruturas vegetais terrestres, sendo as bromélias um dos melhores exemplos deste tipo de ambiente.

As bromeliáceas têm dispositivos bastante diferenciados que permitem a retenção de água entre suas folhas centrais, enquanto os detritos de matéria orgânica vão sendo carreados para a base das folhas mais externas formando assim os tanques laterais. Esta água retida pela bromélia lavou os nutrientes da superfície das folhas situadas em nível superior, bem como recolheu detritos que se misturam nos pequenos tanques. Nestes ambientes, encontramos uma flora e fauna bastante variada, onde é possível observar várias interações ecológicas (Maguire, 1971). De acordo com Margalef (1983), os fitotelmatas estão classificados entre as comunidades aquáticas sob condições de estresse.

A maior parte dos estudos sobre ambientes fitotélmicos está relacionada com a micro e a macrofauna bromelícola (Müller, 1879; Pittendrigh, 1948; Lutz, 1950; Andrade, 1953, 1956; Albuquerque e Lopes, 1976; Hadel e Carvalho, 1988). Além desses trabalhos existem outros com diferentes abordagens (Laessle, 1961; Maguire, 1963, 1971), mas foi Picado (1913) que, ao realizar um 
trabalho fundamental sobre a organização e a fisiologia das bromeliáceas, forneceu os primeiros dados sobre as comunidades fitotelmatas.

$\mathrm{O}$ interesse focalizado neste trabalho sobre o estudo da ficoflórula de ambientes fitotélmicos, surgiu a partir do conhecimento das pesquisas em hidrobiologia sanitária desenvolvidas no Instituto Oswaldo Cruz sobre a biocenose de criadouros naturais de Culex sp. e Anopheles sp., mosquitos responsáveis pela disseminação da malária. Oliveira et al. (1951) destacam a importância dos componentes desta flora fitotélmica, uma vez que foram encontradas diversas microalgas no trato digestivo daquelas larvas.

A bibliografia sobre algas de ambientes fitotélmicos bromelícolas do Estado do Rio de Janeiro limitou-se, até o momento, ao trabalho de Lyra (1971), que estudou as diatomáceas desses ambientes e Nogueira (1991) que registrou pela primeira vez a ocorrência de uma Chlorellaceae em ambiente fitotélmico tropical.

As escassas informações sobre esta flora, motivou o presente estudo, que teve como objetivo inicial inventariar as desmídias deste tipo de ambiente em diferentes pontos do Estado do Rio de Janeiro.

\section{MATERIAL E MÉTODOS}

Ao longo das pesquisas desenvolvidas no Laboratório de Ficologia, Departamento de Botânica, Museu Nacional/UFRJ, iniciadas em 1975, foram reunidas 25 amostras coletadas ao acaso em diversos ambientes fitotélmicos bromelícolas terrestres através do Estado do Rio de Janeiro. Daquele total, 19 continham representantes de desmídias.

As coletas foram efetuadas nos Municípios de Carapebus, Itatiaia, Macaé, Maricá, Niterói, Rio de Janeiro e Saquarema, abrangendo diferentes tipos de hábitat bromelícolas: bromélia de restinga(sol), bromélias de mata(sombra) e bromélias de altitude. As bromélias visitadas são representantes de Aechmea nudicaulis, Bromelia anthiacanta e Neoregelia cruenta.

A retirada da água dos tanques bromelícolas foi realizada utilizando-se uma pipeta de $10 \mathrm{ml}$ com uma pêra de borracha adaptada na extremidade, permitindo assim a sucção da água retida nos imbricamentos das folhas de bromélia e, então, armazenada em frascos plásticos e levadas ao laboratório para exame.
Características como forma do plasto e número de pirenóides só foram descritas quando observadas durante o exame do material.

$\mathrm{Na}$ fixação e preservação do material coletado foi utilizada Solução de Transeau (Bicudo e Bicudo, 1970); o material preservado foi armazenado em frascos de vidro transparentes e estocados no Herbário do Museu Nacional (RJ).

Lista das amostras de algas coletadas nos imbricamentos das folhas de bromélias e que continham desmídias:

R149609 - Município do Rio de Janeiro, BR 101, $\mathrm{Km} \mathrm{18}$, área de restinga (15/10/75).

R149610 - Município do Rio de Janeiro, BR 101, $\mathrm{Km} \mathrm{12}$, área de restinga (13/09/77).

R149877 - Município do Rio de Janeiro, BR 101, $\mathrm{Km} 16$, área de restinga (10/09/77).

R169467 - Município do Rio de Janeiro, BR 101, $\mathrm{Km}$ 9, área de restinga (26/09/76).

R171898 - Município do Rio de Janeiro, Recreio dos Bandeirantes, Pedra do Rangel (12/09/82). R179245 - Município do Rio de Janeiro, BR101, $\mathrm{Km} 8$, área de restinga (10/09/77).

R179315 - Município de Niterói, Piratininga, área de restinga (17/01/83).

R179467 - Município de Maricá, Itaipuaçu, área de restinga (27/09/88).

R179468 - Município de Maricá, Barra de Mariça, entre dunas próximas ao mar (27/09/88).

R179469 - Município de Maricá, Barra de Maricá, entre dunas afastadas do mar (27/09/88).

R179471 - Município de Maricá, Barra de Maricá, proxima à mata de Eugenia sp. (27/09/88).

R179478 - Município de Maricá, restinga de Maricá, área 3, próxima ao mar (15/01/89).

R179479 - Município de Maricá, restinga de Maricá, área 1, próxima ao início da mata (15/01/89). R179480 - Município de Maricá, restinga de Maricá, área 2, região entre o mar e a mata (15/10/89). R179481 - Município de Maricá, restinga de Maricá, área 2, região sombreada por arbustos de maior porte $(15 / 01 / 89)$.

R179482 - Município de Maricá, restinga de Maricá, área 4 , moita numerosa, isolada na parte mediana da restinga (15/01/89).

R179483 - Município de Maricá, restinga de Maricá, área 4, grupo de bromélia terrestres, sem formação de moita (15/01/89). 
R192901 - Município de Macaé, restinga próxima à Lagoa de Cabiúnas (10/11/90).

R192902 - Município de Carapebus, restinga de Carapebus, lado contrário à Lagoa de Carapebus $(12 / 12 / 90)$.

\section{RESULTADOS}

Durante o estudo das comunidades fitotélmicas bromelícolas terrestres do Estado do Rio de Janeiro foram registrados 33 táxons (Tabela 1), distribuídos em seis classes com diferentes contribuições (Fig. 1).

A maior contribuição ficou por conta das Zygnemaphyceae (49\%), destacando-se a Família Desmidiaceae com 14 táxons distribuídos em 7 gêneros, sendo Desmidium grevillii o mais co- mum entre as desmídias estudadas. Chlorophyceae (24\%) estiveram presentes em cerca de $80 \%$ das amostras e dentre elas destacamos Oedogonium itzigsohnii, freqüente nas análises e na maioria dos casos sempre em reprodução sexuada e Scotiellopsis terrestris, espécie antes registrada somente para regiões de clima frio ou temperado, tendo sido citada pela primeira vez em região tropical por Nogueira (1991). Cyanophyceae com uma contribuição de $18 \%$, apresentou predominância de formas filamentosas, tendo sido registrado uma única espécie colonial (Chroococcus sp). Bacillariophyceae, Xanthophyceae e Dinophyceae, representadas cada uma por um único táxon, foram as classes com menor contribuição nos ambientes analisados (Bacillariophyceae + Xanthophyceae + Dinophyceae $=9 \%$ ).

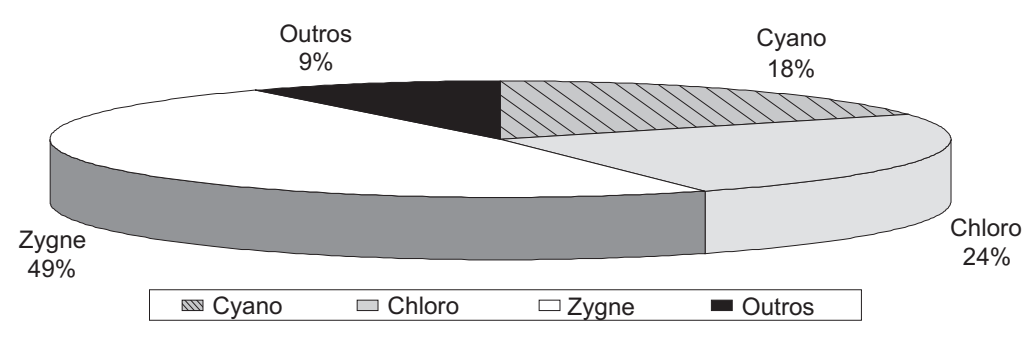

Fig. 1 - Contribuição percentual das classes de algas registradas na composição florística dos ambientes fitotélmicos estudados. $($ Cyano $=$ Cyanophyceae $;$ Chloro $=$ Chlorophyceae Zygne $=$ Zygnemaphyceae Outros $=$ Bacillariophyceae + Xanthophyceae + Dinophyceae).

Cabe ainda ressaltar que as amostras coletadas em locais sombreados não continham representantes de algas; nestas predominaram fungos e muita matéria orgânica particulada, resultante da cobertura vegetal; vez por outra foi registrado a presença de uma microfauna.

As desmídias registradas nos diferentes ambientes bromelícolas estudados estão descritas e ilustradas a seguir:

Zygnemaphyceae

Desmidiales

Desmidiaceae

Cosmarium botrytis (Meneghine) Ralfs var. botrystis

Brit. Desm. p. 99, pl. 16, Fig. 1. 1948.

(Fig. 2)
Células 1,3-1,4 vez mais longas que largas, 6877 x 48-57 $\mu \mathrm{m}$, larg. istmo12-16 $\mu \mathrm{m}$; semicélulas semicirculares, constrição mediana profunda, margens laterais convexas, margem superior estreita, arredondada, parede celular uniformemente granulada.

Material estudado: R179483; R179467.

Distribuição geográfica no Estado do Rio de Janeiro: Duque de Caxias (Oliveira, Andrade e Nascimento, 1951).

A parede celular desta espécie mostrou-se invariavelmente granulosa, sem alteração no arranjo de seus grânulos, diferente da observação feita por Prescott et al. (1981) ao estudar as desmídias norteamericanas.

\section{Cosmarium furcatum Förster}

Hydrobiologia 23(3/4): 391. 1964.

(Figs. 3 e 4 ) 
Células 1,1 vez mais largas que longas, 34$40 \times 39-45 \mu \mathrm{m}$, larg. istmo $10-12 \mu \mathrm{m}$; semicélulas subtriangulares, constrição mediana profunda, aberta; margens laterais divergentes com espinhos em toda extensão; margem superior amplamente côncava, com espinhos em toda a parede, bifurcados nos ângulos; vista vertical elíptica.

Material estudado: R179471; R179483.

Distribuição geográfica no Estado do Rio de Janeiro: primeira citação da ocorrência da espécie.

Esta espécie foi descrita pela primeira vez por Förster para o Estado de Goiás. As medidas apresentadas por Förster (1964) correspondem na realidade à Fig. 26 da pl. 22. Enquanto as Figs. 27 e 28 , da mesma prancha, apresentam as medidas dentro dos limites métricos registrados para o material fitotélmico.

Cosmarium obtusatum Schmidle var. obtusatum Engler's Bot. Jahrb. 26(1): 38. 1898.

(Figs. 5 e 6)

Células 1.1-1.5 vez mais longas que largas, 48-58 x 32-48 $\mu \mathrm{m}$, larg. istmo 9-14 $\mu \mathrm{m}$; semicé- lulas piramidal-truncadas, constrição mediana profunda, ângulos basais arredondados, margem apical truncada, levemente ondulada; vista lateral arredondada, margens laterais onduladas; vista vertical elíptica alargada, pólos ondulados; 2 pirenóides por semicélula.

Material estudado: R149609; R169467; R1 79468; R179467.

Distribuição geográfica no Estado do Rio de Janeiro: primeira citação da ocorrência da variedade.

Ruzicka (1953) faz ampla discussão sobre a referida espécie, suas variedades e formas, além das espécies próximas, que podem ser confundidas com C. obtusatum.

Cosmarium speciosum Lundel var.simplex

Nordstedt f. simplex

Öfv. Kongl. Vet-Akad. Förhandl. 1872(6): 31, pl. 6, Fig. 12. 1872.

(Figs. 7-9)

Células 1,3-1,5 vez mais longas que largas, 40-46 x 27-33 $\mu \mathrm{m}$, larg. istmo 10-12 $\mu \mathrm{m}$; semicélulas campanuladas, constrição mediana profunda,

TABELA 1

Relação dos táxons, em suas respectivas classes, registrados nos ambientes fitotélmicos bromelícolas estudados.

\begin{tabular}{l}
\hline CYANOPHYCEAE \\
Anabaena sp. \\
Chroococcus sp. \\
Lyngbya sp. \\
Oocystis sp. \\
Oscillatoria sp. \\
Schizothrix sp \\
CHLOROPHYCEAE \\
Apodochloris sp. \\
Chlorela sp. \\
Eutetramorus planctonicus \\
Microspora sp. \\
Oedogonium itzigsohnii \\
Scenedesmus sp. \\
Scotiellopsis terrestris \\
Ulothrix sp. \\
ZYGNEMAPHYCEAE \\
Cosmarium botrytis \\
C. furcatum \\
C. obtusatum \\
\hline
\end{tabular}

Cosmarium speciosum var. simplex

Demidium grevillii

D. quadratum

Euastrum denticulatum

Mougeotia sp.(estéril)

Pleurotaenium ehrenbergii

P. trabecula var. elongatum

Spirogyra sp. (estéril)

Staurastrum anatinum var. anatinum f. parvum

S. chaetoceras

S. hirsutum

Staurodesmus triangularis var. subparallelus

Xanthidium concinum

BACILLARIOPHYCEAE

Tabelaria $\mathrm{sp.}$

XANTHOPHYCEAE

Tribonema sp.

DINOPHYCEAE

Peridinium umbonatum 
margem da semicélula crenulada, cerca de 21 crenulações, parede celular com grânulos ausentes no centro da semicélula; vista lateral ovada, ápice amplamente arredondado; vista vertical elíptica, alargada na região mediana, pólos crenulados; 1 pirenóide por semicélula.

Material estudado: R179315.

Distribuição geográfica no Estado do Rio de Janeiro: primeira citação da ocorrência da forma.

\section{Desmidium grevillii (Kützing ex Ralfs) De Bary} Conjugatae 42, pl. 4, Figs. 30-31. 1858.

(Figs. 10 e 11)

Filamentos torcidos, células 2,3-2,6 vezes mais largas que longas, 22-27 x 52-72 $\mu \mathrm{m}$, larg. istmo $48-63 \mu \mathrm{m}$; semicélulas sub-retangulares, margens laterais convexas, margem apical amplamente truncada, parede celular lisa; vista vertical elíptica com 1 mamilo em cada pólo; cloroplastídio axial, 6-lobado.

Material estudado: R149877; R171898; R1 79469; R179478; R179479; R179480; 179481; R179482; R179483.

Distribuição geográfica no Estado do Rio de Janeiro: Duque de Caxias (Oliveira, Andrade \& Nascimento, 1951; Andrade, 1953, 1956); Silva Jardim (Sophia, 1984, como D. cylindricum Greville ex Nordstedt var. cylindricum) e Rio de Janeiro (Sophia, 1987).

\section{Desmidium quadratum Nordstedt var. quadratum}

Acta Univ. Lund 9: 49. pl. 1, Fig. 24. 1873.

(Figs. 12 e 13)

Filamento torcido; células 1,1-1,2 vez mais largas que longas, 19-21 x 22-23 $\mu \mathrm{m}$, larg. ápice 17-22 $\mu \mathrm{m}$, semicélulas trapezoidal-truncadas, constrição mediana pouco marcada, margens laterais com uma intumescência basal arredonda$\mathrm{da}$, convergente na parte superior; margem superior amplamente truncada, parede celular lisa; vista vertical subcircular com 1 mamilo em cada pólo; cloroplastídio axial, 6-lobado.

Material estudado: R149609; R149610.

Distribuição geográfica no Estado do Rio de Janeiro: Município do Rio de Janeiro (Sophia, 1987).

De acordo com Bicudo e Samanez (1984) algumas citações desta espécie são problemáticas e, ainda, segundo estes mesmos autores, a pare- de celular do táxon em questão é composta de 3 séries transversais paralelas de pontuações; no entanto, o material examinado por nós apresentou sempre a parede celular lisa, semelhante àquele citado e ilustrado em Scott et al. (1965) para o Estado do Pará.

\section{Euastrum denticulatum (Kirch) Gay}

Bull. Soc. Bot. France 31: 335. 1884.

(Figs. 14-16)

Células 1,2-1,3 vez mais longas que largas, 24-30 x 19-22 $\mu \mathrm{m}$, larg. lobo apical 12-17 $\mu \mathrm{m}$, larg. istmo 3-5 mm, semicélulas piramidal-truncadas, margens laterais lobadas, depois côncavas em direção ao ápice, 1 espinho em cada lobo, ápice reto, com uma incisão mediana bem marcada, ângulos externos com 1 espinho de cada lado; parede celular com 1 dentículo intramarginal na direção de cada ângulo, decoração facial presente; vista lateral oval, pólos truncados; vista vertical elíptica alargada, pólos arredondados.

Material estudado: R149877.

Distribuição geográfica no Estado do Rio de Janeiro: primeira citação da ocorrência da espécie.

Esta espécie é bastante confusa e carece urgente de uma revisão para se estabelecer melhor seus limites e suas variedades. Inicialmente, pode ser confudida com E. pseudocoralloides Fritch, como ilustrado por Prescott et al. (1977: 243, Fig. 250 ), no qual a diferença está na margem lateral do lobo polar que em E. pseudocoralloides é levemente projetada e truncada. Também pode ser confundida com o grupo dos E. abruptum, principalmente a var. subglaziovii Krieger, da qual difere por apresentar, esta última, 3 pequenos grânulos em cada ângulo dos lobos laterais.

\section{Pleurotaenium ehrenbergii (Brébisson) De Bary} var. eherenbergii

Untersuch. Fam. Conjug. p. 75. 1858.

(Figs. 17-19)

Células 17 vezes mais longas que largas, 400$480 \times 26-28 \mu \mathrm{m}$, larg. istmo $22-25 \mu \mathrm{m}$; larg. ápice: 21-25 $\mu \mathrm{m}$; constrição mediana evidente; semicélulas subcilíndricas, margens laterais onduladas, paralelas, pouco atenuadas em direção ao ápice amplamente truncado-arredondado, 3 grânulos visíveis abaixo da margem superior; parede celular grosseiramente pontuada; cloroplastídios fitáceos, parietais, numerosos pirenóides ao longo de cada fita. 


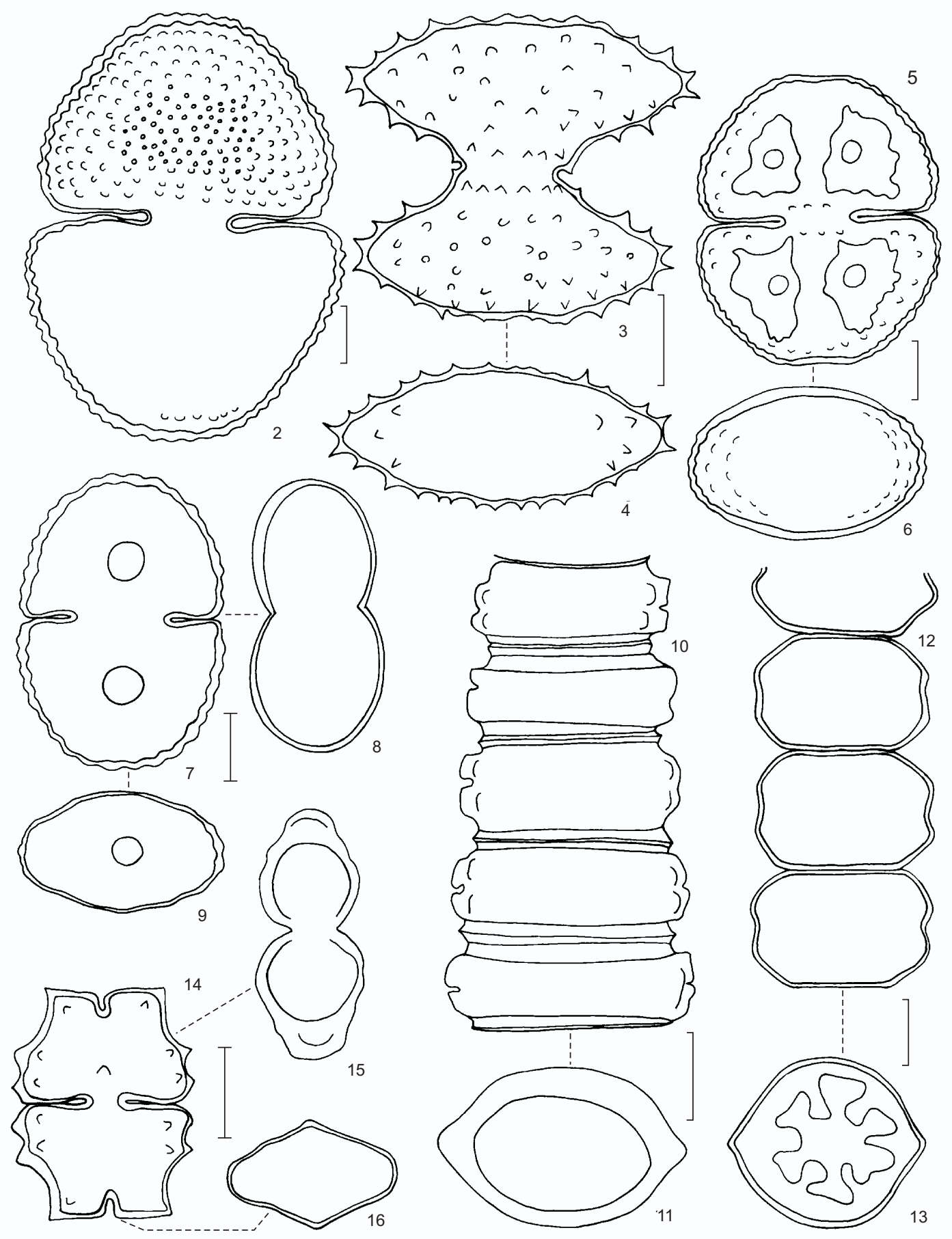

Figs. 2-16 - Fig. 2 - Cosmarium botyitis var. botrytis; Figs. 3 e 4 -Cosmarium furcatum; Figs. 5 e 6 - Cosmarium obtusatum var. obtusatum; Figs. 7-9 - Cosmarium speciosum var. simplex f. simplex; Figs. 10 e 11 - Desmidium grevillii; Figs. 12 e 13 Desmidium quadratum var. quadratum; Figs. 14-16 - Euastrum denticulatum. (Escalas: Figs. 10 e $11=25 \mu \mathrm{m}$; demais $=10 \mu \mathrm{m}$ ). 
Material estudado: R192901.

Distribuição geográfica no Estado do Rio de Janeiro: Rio de Janeiro (Borge, 1899)

Pleurotaenium trabecula (Ehrenberg) ex Nägeli var. elongatum Cedergren

Ark. f. Bot. 13(4): 1-43. 1913.

(Figs. 20-22)

Células 18-22 vezes mais longas que largas, 494-550 x 24-28 $\mu \mathrm{m}$, larg. istmo $20-25 \mu \mathrm{m}$; larg. ápice 18-22 mm; constrição mediana evidente; semicélulas subcilíndrica, margens laterais com uma intumescência basal, atenuadas em direção ao ápice, ápice truncado-arredondado, liso, parede celular pontuada; cloroplastídios 4 , parietais, fitáceos, numerosos pirenóides ao longo de cada fita.

Material estudado: R149877; R179245.

Distribuição geográfica no Estado do Rio de Janeiro: Duque de Caxias (Andrade, 1956); Rio de Janeiro (Sophia, 1991).

Staurastrum anatinum var. anatinum $f$. parvum (West) Prescott, Bicudo \& Vinyard.

In Prescott et al., A synopsis of North American desmids, 2: Desmidiaceae: Placodemae, 4: 123. 1982.

(Figs. 34 e 35)

Células 1,2 vez mais largas que longas (com processos), 18-22 x 24-26 $\mu \mathrm{m}$ (com processos); larg. istmo 3-5 $\mu \mathrm{m}$; semicélulas copuliformes, margens laterais divergentes, prolongadas em três processos longos; margem apical côncava; vista vertical triangular, margem da célula com espinhos.

Material estudado: R179483.

Distribuição geográfica no Estado do Rio de Janeiro: Araruama, Lagoa de Juturnaíba (Lima, 1982; Huszar et al., 1989, como St. paradoxum var. parvum).

Brook (1959,1959a), ao realizar um estudo sobre as populações de St anatinun, observou várias similaridades entre St. paradoxum, St. gracile, outras espécies afins e suas respectivas variedades, e afirma ser inaceitável a espécie tipo de St. paradoxum. O referido autor ao comparar as descrições e ilustrações de diferentes pesquisadores notou que são muito semelhantes e, então, considera St. paradoxum como f. paradoxum de St. anatinum, por ser esta última a mais antiga. Deste modo, Prescott et al. (1982) propõe uma nova combinação para a f. parva de St. paradoxum: St. anatinum var. anatinum $f$. parvum
Staurastrum chaetoceras (Schoeder) G. M. Smith Wisconsin Geol. \& Nat. Hist. surv. Bull. 57(2): 99, pl. 76, Figs. 21-24; pl..77, Fig. 1. 1924.

(Figs. 27 e 28)

Células 2,4-2,5 mais largas que longas (incluindo os espinhos), 35-40 x 89-96 $\mu \mathrm{m}$ (com processos); 24-26 x 17-20 $\mu \mathrm{m}$ (sem processos), larg. istmo 5-7 $\mu \mathrm{m}$, semicélulas inversamente triangulares, margem apical elevada na parte mediana; vista vertical fusiforme-alargada, pólos prolongados em processos longos.

Material estudado: R179483.

Distribuição geográfica no Estado do Rio de Janeiro: primeira citação da ocorrência da espécie.

O material descrito por Prescott et al. (1982) está caracterizado pela presença de grânulos na região do istmo, enquanto Smith (1924), obra original para esta espécie, ao estudar o fitoplâncton de Wisconsin, refere-se à presença dos referidos grânulos como facultativos, podendo estar presentes ou não.

No material estudado não foram observados grânulos na região pré-istmal; nossos exemplares apresentaram também uma curvatura menor nos processos das semicélulas.

Staurastrum hirsutum (Ehrenberg) Ralfs

Brit. Desm. p. 127, pl. 22, Fig. 3. 1848.

(Figs. 23-26)

Células 1,2-1,5 vez mais longas que largas (incluindo os espinhos) 37-54 x 30-36 $\mu \mathrm{m}$, larg. istmo 6-8 $\mu \mathrm{m}$; semicélula oval, constrição mediana profunda, aberta; margens laterais e apical amplamente convexas; parede celular com espinhos em séries concêntricas, ausentes na região do istmo; vista vertical 3-4 angular, margens convexas, espinhos presentes em toda a parede celular.

Material estudado: R149877; R171898; R1 79469; R192901.

Distribuição geográfica no Estado do Rio de Janeiro: Rio D'ouro (Nova Iguaçu, Wille, 1884).

Material extremamente difícil de ser trabalhado, uma vez que pode ser confundido com: St. teliferum Ralfs e St. echinatum Brébisson. Existe a necessidade de uma profunda revisão nestes referidos grupos de algas, a fim de estabelecer melhor os limites taxonômicos das diferentes espécies citadas.

A identificação do material fitotelmata como St. hirsutum (Ehrenberg) Ralfs ficou por conta da relação comprimento/largura da célula, a forma 
da semicélula, istmo estreito, tamanho e arrumação dos espinhos, bem como a convexidade da parede celular em vista vertical, nos indivíduos 3-angulares.

Em uma amostra populacional examinada (R192901) registramos indivíduos de vista apical 4-angular (Figs. 25 e 26). Pelo fato de possuírem o mesmo padrão de decoração, estarem dentro do intervalo de medidas fornecido pela literatura especializada e ocorrerem na mesma amostra, sugerimos que esta espécie englobe também os indivíduos de vista apical 4-angular.

Staurodesmus triangularis (Lagerheim) Teiling var. subparallelus (G.M. Smith) Teiling

Bot. Notiser 1948(1): 62, Figs. 67 e 68. 1948.

(Figs. 32 e 33)

Células 2,3-2,7 vezes mais largas que longas, com espinho, $18-20 \times 43-52 \mu \mathrm{m}$; larg. istmo 3-5 $\mu \mathrm{m}$; semicélulas trapezoidais, margens laterais e basal retas, ângulos prolongados em um longo espinho horizontal; vista apical elíptica, cada pólo com um espinho longo, reto; parede celular lisa.

Material estudado: R179483.

Distribuição geográfica no Estado do Rio de Janeiro: primeira citação da ocorrência da variedade.

\section{Xanthidium concinum Archer}

Ann. Mag. Nat. Hist. 5(11): 285. 1883.

(Figs. 29-31)

Células tão longas quanto largas, incluindo os espinhos, $12-13 \times 12-13 \mu \mathrm{m}$, larg. istmo 2-4 $\mu \mathrm{m}$; constrição mediana profunda, semicélulas subhexagonal, margem lateral angulosa, com 1 espinho, margem apical amplamente truncada com 1 espinho reduzido em cada ângulo; face central da semicélula com 1 espinho na parte mediana da semicélula, um de cada lado da parede celular; vista vertical elíptica, com 1 espinho em cada pólo e 1 espinho reduzido entre cada espinho maior; 1 pirenóide por semicélula.

Material estudado: R192902.

Distribuição geográfica no Estado do Rio de Janeiro: primeira citação da ocorrência da espécie.

Agradecimentos - A Bruno Pedrosa, estagiário de Iniciação Científica do Laboratório de Ficologia, Depto de Botânica, M.N./UFRJ, pela colaboração em várias etapas deste trabalho e a Profa. Lúcia Helena Sampaio da Silva, do mesmo Laboratório, pelas valiosas sugestões apresentadas.

\section{BIBLIOGRAFIA}

ALBUQUERQUE, I. R. S. \& LOPES, S. M. R., 1976, Blataria de Bromélia (Dictyoptera). Rev. Brasil. Biol. 36(4): 873 901 .

ANDRADE, R. M., 1953, Observações hidrobiológicas sobre o Anopheles tarsimaculatus: I. Relações com alguns organismos planctônicos. Rev. Brasil. Malar. Doenç. Trop., Rio de Janeiro, 5(1): 95-107.

ANDRADE, R. M., 1956, Observações hidrobiológicas sobre o Anopheles tarsimaculatus: III. Distribuição, freqüência de ocorrência e densidade relativa de organismos planctônicos em alguns de seus biótopos. Rev. Brasil. Malar. Doenç. Trop., Rio de Janeiro, 8: 443-490.

BICUDO, C. E. M. \& BICUDO, R. M. T., 1970, Algas de águas continentais brasileiras: chave ilustrada para identificação de gêneros. São Paulo: Editora da Universidade de São Paulo, 228p.

BICUDO, C. E. M. e SAMANEZ, I. M., 1984, Desmidioflórula Paulista III. Bibliotheca Phycologica, 68: 1-139.

BORGE, O., 1899, Ueber tropische und subtropische süsswasser. Chlorophyceen. Bih. K. svenska Vetenskaakad. Handl., Stokholm, 24(12): 1-33.

BROOK, A. J., 1959, De Brébisson's determinations of Staurastrum paradoxum Meyen and S. gracile Ralfs. Nova Hedwigia, 1(2): 163-165.

BROOK, A. J., 1959a , Staurastrum pendulum var. pinguiforme Croasdale, S. minor West f. major, f. nov., fac. quadrata and S. micron var. perpendiculatum (Gronblad) nov. comb., desmids new to the British freshwater plankton. Nova Hedwigia, 1(2): 157-162.

FÖRSTER, K., 1964, Desmidiaceen aus Brasilien z. Teil: Bahia, Goyaz, Piauhy und Nord-Brasilien. Hydrobiologia, 23(3/4): 321-505.

HADEL, V. F., 1989, A fauna associada aos fitotelmata bromelícolas da Estação Ecológica da Juréia - Itatins (SP). Dissertação de Mestrado, Universidade de São Paulo, 128p.

HADEL, V. F. \& CARVALHO, M. A. J., 1988, Associação copépodo/bromélia: relação com o tipo de tanque. Acta Limnol. Brasil., 2: 571-586.

HUSZAR, V. L. M., MENEZES, M. \& FERNANDES, V. O., 1989, Fitoplâncton de rede da Lagoa Cabiúnas, Macaé, Estado do Rio de Janeiro, Brasil: uma contribuição a seu conhecimento. Bol. Mus. Nac., nov. ser., Rio de Janeiro, 78: 1-43.

LAESSLE, A. M., 1961, A micro-limnological study of Jamaican Bromeliads. Ecology, 42: 499-517.

LIMA, M. G. S. M., 1982, Desmidiaceae (Zygnemaphyceae) do Município do Rio de Janeiro: uma contribuição ao seu conhecimento. Dissertação de Mestrado, Universidade Federal do Rio de Janeiro, 174p.

LYRA, L. T., 1971, Algumas diatomáceas encontradas em Bromeliáceas, Brasil. Mem. Inst. Oswaldo Cruz, Rio de Janeiro, 69(1): 129-139.

LUTZ, A., 1950, Mosquitos da floresta e malária silvestre. Rev. Brasil. Malar., 2(2): 91-99. 


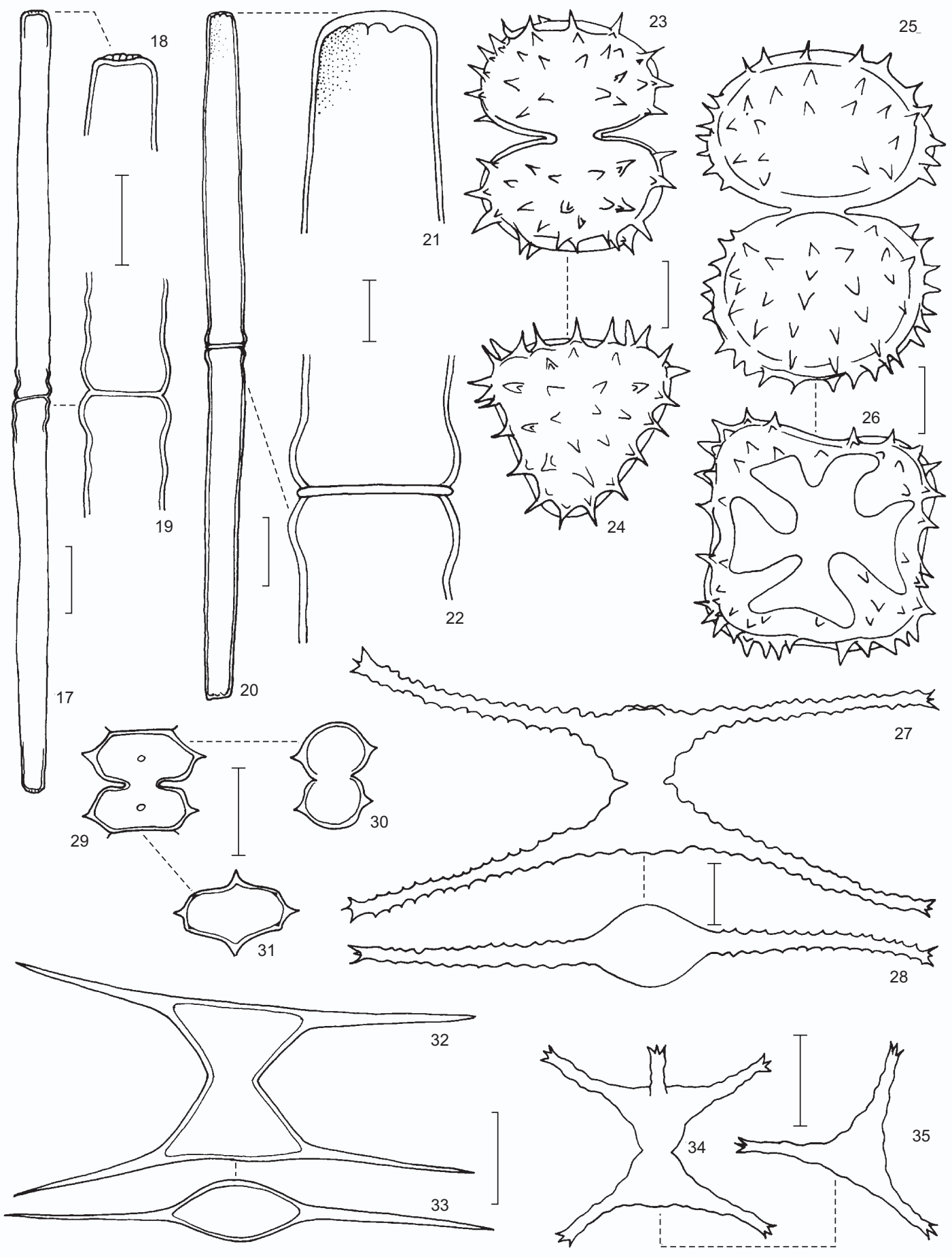

Figs. 17-35 - Figs. 17-19 - Pleurotaenium eherenbergii var. eherenbergii; Figs. 20-22 - Pleurotaenium trabecula var. elongatum; Figs. 23-26 - Staurastrum hirsutum; Figs. 27 e 28 - Staurastrum chaetoceras; Figs. 29-31 - Xanthidium concinum; Figs. 32 e 33 - Staurodesmus triangularis var. subparallelus; Figs. 34 e 35 - Staurastrum anatinum var. anatinum f. parvum. (Escalas: Figs. $17-20=50 \mu \mathrm{m} ; 18$ e $19=25 \mu \mathrm{m}$; demais: $10 \mu \mathrm{m}$ ). 
MAGUIRE, B. Jr., 1963, The passive dispersal of small aquatic organisms and there colonization of isolated bodies of water. Ecol. Monogr, 33(2): 161-185.

MAGUIRE, B. Jr., 1971, Phitotelmata: Biota and community structure determination in plant-held water. An. Rev. Ecol. Syst., Palo Alto, 2: 439-464.

MARGALEF, R., 1983, Ecologia. Editora Omega, 951pp.

MÜLLER, F, 1879, Descrição do Elpidium bromeliarum crustáceo da Família dos Cytherideos. Archos Mus. nac., Rio de Janeiro, 1879: 27-34.

NOGUEIRA I. S., 1991, Primeiro registro de ocorrência de Scotiellopsis terrestris (Chlorellales, Chorellaceae ) em ambiente fitotélmico tropical. Rev. Brasil. Biol., 51(2): 437-444.

OLIVEIRA, L., ANDRADE, R. M. \& NASCIMENTO, R., 1951, Contribuição ao estudo hidrobiológico dos criadouros do Anopheles Tarsimaculatus Goeldi, 1905 (= Anopheles aquasalis Curry, 1932) na Baixada Fluminense. Rev. Brasil. Malar Doenç. Trop 3(2): 151-226.

PICADO, C., 1913, Le bromeliaceés epiphytes considerées comme milieu biologique. Bull.scient.France et Belgique, 47: 215-360.

PITTENDRIGH, C. S., 1948, The bromeliad-anophelesmalaria complex in Trinidad I - The Bromeliad flora. Evolution, 2: 58-89.

PRESCOTT, G. W., BICUDO, C. E. M., VINYARD, W. C., 1982, A synopsis of North American Desmids, II. Desmidiaceae: Placodermae, Sec. 4. Lincoln and London Ed., 700p.

PRESCOTT, G. W., CROASDALE, H. T. \& VINYARD, W. C, 1977, A synopsis of North American Desmids, II. Desmidiaceae: Pacodermae, Sec. 2. Lincoln and London Ed., $413 \mathrm{p}$.
PRESCOTT, G. W., CROASDALE, H. T., VINYARD, W. C. \& BICUDO, C. E. M., 1981, A synopsis of North American Desmids, II. Desmidiacea: Placodermae, Sec. 3. Lincoln and London Ed., 720p.

RUZICKA, J., 1953, Cosmarium obtusatum Schmidle. Preslia, 25: 229-262.

SCOTT, A. M., GRÖNBLAD, R. \& CROASDALE, H., 1965, Desmids from the Amazon Basin, Brazil, collected by Dr. H. Sioli. Acta Bot. Fenn., 69: 3-93.

SMITH, G. M., 1924, Phytoplankton of the Inland Lakes of Wisconsin. 2. Desmidiaceae. Bull. Wis. Geol. Nat. Hist. Surv., 57: 1-227.

SOPHIA, M. G., 1984, Contribuição ao conhecimento da flora ficológica da Lagoa de Juturnaíba, R.J., Brasil: Desmidiaceae (Zygnemaphyceae) de hábito filamentoso. Bradea, Rio de Janeiro, 4(13): 81-90.

SOPHIA, M. G., 1987, Contribuição ao conhecimento das desmídias de hábito filamentoso do Estado do Rio de Janeiro, Brasil. Rickia, 14: 21-35.

SOPHIA, M. G., 1991, Desmídias de hábito solitário (exceto Micrasterias C. Agardh ex Ralfs) do Município do Rio de Janeiro e arredores, Brasil. Rev. Brasil. Biol., 51(1): 85-107.

VARGA, L., 1928, Ein interessanter Biotop der Biocönose von Wasserorganismen. Biol. zentralblatt, 48: 143-162.

WILLE, N., 1884, Bidrag til sydamerikas algflora. I-III. Bir. Kongl. Svenska Vet.-AKad. Handl. 8(13): 1-64. 